\title{
Radiation therapy for primary central nervous system lymphoma
}

\author{
Yuta Shibamoto \\ Department of Radiology, Nagoya City University Graduate School of Medical Sciences, Nagoya, \\ Japan
}

\begin{abstract}
Up until the late 1970s, radiation therapy played an important role in the treatment of primary central nervous system lymphoma (PCNSL) but more recently its role has changed due to the increased use of systemic chemotherapy. In this article, the current status of radiotherapy for PCNSL and optimal forms of radiotherapy, including the treatment volume and radiation dose, are discussed. Data from nationwide Japanese surveys of PCNSL patients treated with radiation therapy suggest that the prognosis of PCNSL patients improved during the 1990 s, in part due to the use of high-dose methotrexate-containing chemotherapy. The prognosis of patients treated with radiation alone also improved. Radiotherapy still seems to play an important role in the attempt to cure this disease.
\end{abstract}

\section{Introduction}

Primary central nervous system lymphoma (PCNSL) responds relatively quickly to radiation therapy, like the majority of extra-central nervous system (CNS) lymphomas. The complete disappearance of enhancing tumor masses is frequently observed following conventional radiation therapy. Therefore, until the late 1970 s, radiation alone after surgical resection was the standard treatment for PCNSL. However, the outcome of patients treated by radiation alone was generally poor. ${ }^{1-5}$ In particular, older studies reported 5 -year survival rates

Correspondence: Yuta Shibamoto, Department of Radiology, Nagoya City University Graduate School of Medical Sciences, 1 Kawasumi, Mizuho-cho, Mizuho-ku, Nagoya 467-8601 Japan.

Tel: (+81)52-853-8274 - Fax: (+81)52-852-5244.

E-mail: yshiba@med.nagoya-cu.ac.jp

Key words: brain neoplasm, lymphoma, central nervous system, radiotherapy, chemotherapy.

Conflict of interests: the author declares no potential conflict of interests.

Received for publication: 22 July 2013.

Revision received: Not required.

Accepted for publication: 26 August 2013.

This work is licensed under a Creative Commons Attribution NonCommercial 3.0 License (CC BY-NC 3.0).

@C Copyright Y. Shibamoto, 2013

Licensee PAGEPress, Italy

Oncology Reviews 2013; 7:e4

doi:10.4081/oncol.2013.e4 of less than $10 \%$. This poor outcome was in striking contrast with that reported for other extranodal localized (stage I) lymphomas. ${ }^{6,7}$ Although the initial response of PCNSL to radiation is generally good, local recurrence in the irradiated volume, as well as remote CNS recurrence outside of the treatment volume, is frequently observed. This led to changes in the treatment strategy, including the introduction of systemic chemotherapy in the late 1970 s. Combination with standard chemotherapy regimens used for systemic lymphoma did not yield remarkable results. ${ }^{8-11}$ However, subsequently, high-dose methotrexate (MTX)-containing regimens were found to be effective and their use has spread worldwide. ${ }^{12-21}$ However, there is a big flaw in this increase in the routine use of MTX-containing chemotherapy: the lack of firm evidence demonstrated by randomized trials. Even without phase III studies, there has been a recent and growing trend in Western countries to use chemotherapy first and reserve radiation therapy until recurrence. However, approaches like these have not been generally accepted by the international scientific community. It can, therefore, be concluded that the standard treatment for PCNSL has not yet been established because of the paucity of reliable randomized clinical trials. In Japan, radiation therapy is still used in the firstline treatment of non-elderly patients with PCNSL in combination with chemotherapy, generally using MTX-containing regimens. This review summarizes the results of radiation therapy for PCNSL, characterizes its role, and discusses optimal forms.

\section{Strategy for diagnosis}

Central nervous system lymphoma (CNSL), either primary or secondary, showing typical imaging characteristics is not hard to diagnose using contrast-enhanced magnetic resonance imaging (MRI) or even computed tomography (CT). ${ }^{10}$ Typically, CNSL shows marked homogenous contrast enhancement surrounded by broad low-intensity areas on T1-weighted MR images, high-intensity areas on T2weighted images, and low-density areas on CT. The mass effect is, however, relatively small even in a large tumor. It is not difficult to diagnose typical cases from these imaging findings. In addition, if corticosteroids are used and there is a resultant decrease in tumor volume (although steroids are not recommended before biopsy), the clinical diagnosis of CNSL is firmly supported. If the patient has extra-CNS histologically-verified lymphoma, biopsy of the CNS tumor possessing these imaging characteristics is unnecessary and should be avoided. Therefore, when imaging findings suggest CNSL, positron emission tomography with 18-fluorodeoxyglucose is highly recommended to evaluate the existence of extra-cranial lymphoma. If there is no extraCNS lesion, a diagnosis of PCNSL becomes very likely. Nevertheless, the author recommends biopsy of the suspected PCNSL. Extensive resection is not recommended, since it has not been proven to improve the prognosis. ${ }^{22}$

The recommendation to perform a biopsy is based on the fact that, 
according to the current classification of lymphoma, most PCNSL are categorized as diffuse large B-cell lymphoma. However, a small proportion of PCNSL are of T-cell origin, and there are other types of B-cell lymphoma in PCNSL. ${ }^{22}$ Differentiating between the various histological subtypes of lymphoma by imaging is problematic. More importantly, in the future, the classification of lymphoma may change again since the present classification does not necessarily reflect the origin of lymphoma cells. Given this, it could be meaningful to obtain a biopsy and establish the histopathological diagnosis, and then store the specimen for future reference.

\section{Treatment strategy}

In view of the poor results obtained by radiation therapy alone, combination with chemotherapy has been investigated since the late 1970s. Most regimens of chemotherapy investigated so far can be classified into the following two groups: i) regimens used for systemic lymphoma, such as CHOP (cyclophosphamide, doxorubicin, vincristine, and prednisolone); and ii) high-dose MTX either alone or in combination with other drugs. The outcome of patients treated by the former regimens was mostly unsatisfactory. ${ }^{9-11}$ A small randomized study suggested no benefit of adding CHOP chemotherapy to whole-brain radiation. ${ }^{11}$ These unsatisfactory results were attributed to poor penetration of the compounds across the blood-brain barrier, especially when given before radiation therapy. However, in a previously published paper, our group ${ }^{10}$ suggested that the use of CHOP or CHOP-like chemotherapy after radiation therapy might improve outcome, since radiation therapy can disrupt the blood-brain barrier. ${ }^{23}$ The drugs that do not cross the blood-brain barrier may cross it when given after radiation therapy. This hypothesis, however, still has to be examined in greater depth.

In contrast, studies investigating high-dose MTX-containing regimens appear to have been successful. ${ }^{12-21}$ Many such studies achieved median survival times of 30-45 months and 5-year survival rates of 30 $40 \%$. However, no randomized studies comparing radiation alone with radiation plus high-dose MTX-based chemotherapy have been carried out. The investigators of chemoradiation trials have reported that the outcome obtained is a marked improvement over the results previously achieved by radiotherapy alone (i.e. 5 -year survival $<10 \%$ ). Even in the absence of randomized studies, some investigators report that such chemoradiation therapy is definitely superior to radiation alone. ${ }^{24}$ However, this methodology (i.e. comparison with very old data obtained with radiation alone) does not seem to be justified in the era of evidence-based medicine.

Nevertheless, attempts to defer radiation therapy in elderly patients and reserve radiation for recurrence after primary chemotherapy in younger patients seem to be steadily on the increase. Chemotherapy alone is associated with a higher recurrence rate but it has been reported to be associated with less neurotoxicity. A recent randomized trial comparing high-dose MTX alone and in combination with radiation therapy clearly indicated a lower progression-free survival in patients undergoing chemotherapy alone (see below). ${ }^{25}$ The optimal treatment policy will need to be established in the near future.

\section{Radiation therapy}

\section{Results of radiotherapy alone}

Table 1 summarizes reported results of radiation monotherapy for PCNSL. Results of old small-scale studies are included in two reviews. ${ }^{1,2}$ Relatively old literature indicates a poor outcome for PNCSL
Table 1. Results of radiation monotherapy for primary central nervous system lymphoma.

\begin{tabular}{|c|c|c|c|}
\hline $\begin{array}{l}\text { First author } \\
\text { (Group) }\end{array}$ & $\begin{array}{c}\text { Year } \\
\text { publication no.) }\end{array}$ & $\begin{array}{l}\text { Median survival } \\
\text { (months) }\end{array}$ & $\begin{array}{r}\text { 5-year survival } \\
(\%)\end{array}$ \\
\hline Littman (Review) & $975(150)$ & - & 6 \\
\hline Leibel (Review) & 1987 (114) & 24 & 7 \\
\hline Shibamoto (Kyoto) & $1990(16)$ & 8 & 6 \\
\hline Nelson (RTOG) & $1992(41)$ & 12 & 28 (at 2 years) \\
\hline Watne (Norway) & $1992(15)$ & 19 & 13 \\
\hline Ishikawa (Gunma) & $2003(25)$ & $13^{*}$ & 35 \\
\hline Shibamoto (JASTRC & 20) 2005 (132) & 18 & 18 \\
\hline
\end{tabular}

Kyoto, Kyoto University Hospital; RTOG, Radiation Therapy Oncology Group; Norway, Norwegian Radium Hospital; Gunma, Gunma University Hospital; JASTRO, Japanese Society for Therapeutic Radiology and Oncology CNS Lymphoma Study Group. *For 33 patients, including 8 patients undergoing systemic chemotherapy without methotrexate.

patients treated by radiation alone; the reported 5 -year survival rates are mostly less than $10 \%$. A very high proportion of PCNSL show local recurrence. Although recurrence within the CNS apart from the primary lesion is also common regardless of the radiation treatment volume, ${ }^{5,26}$ in-field local recurrence is often observed. Therefore, it is argued that the radiosensitivity of PCNSL may be lower than that of other extranodal lymphomas. This relatively low radiosensitivity may be intrinsic to PCNSL cells or may be due to the tumor bed (i.e. brain parenchyma). Similarly, CNS germinomas are known to require higher radiation doses for local control than their testicular counterparts (i.e. seminomas). ${ }^{27}$ Nevertheless, the previously reported 5 -year survival rates of less than $10 \%$ appear to be too low for PCNSL that does not frequently metastasize to extra-CNS organs.

The main reason for the poor results in the past is not clear. However, experience shows that before 1980, the diagnosis of PCNSL was not readily made, and the treatment policy for PCNSL patients was not well established. The surgical approach for some of our patients was excessive, leading to a poor post-operative performance status and death soon after. Our group ${ }^{22}$ recently reported that the proportion of good performance status (PS) patients was significantly higher in more recent years than it had been previously. With the development of a diagnostic strategy and better recognition of PCNSL on the part of physicians, patients may now be diagnosed at an earlier stage than before. We speculate that improvement in general care and an earlier diagnosis may be some of the reasons for the improved prognosis.

On the other hand, our group reported a 5 -year survival of $18.0 \%$ in Japanese patients treated by radiotherapy alone between 1990 and $1999 .^{28}$ Patient data were collected through retrospective multi-institutional studies of the Japanese Society for Therapeutic Radiology and Oncology (JASTRO) Lymphoma Study Group and the Chubu Radiation Oncology Group (CROG). ${ }^{29-31}$ It should be noted that there was a gradual increase in the use of combination chemotherapy through the 1990 s, and many of these patients were considered not to be candidates for chemotherapy or refused this treatment approach. Nevertheless, survival data were better than those reported before 1990. In order to compare with chemoradiation studies, 62 patients aged 16-65 years with PS 0-3 were also analyzed in the study. ${ }^{28}$ In terms of age and PS, these patients were considered eligible for a prospective study of chemoradiation by the European Organization for Research and Treatment of Cancer (EORTC 20962) ${ }^{20}$ For these 62 patients, the median survival time was 26 months, and survival rates were $69 \%$ at 1 year, $53 \%$ at 2 years, $33 \%$ at 3 years, and $24 \%$ at 5 years. For comparison, in the EORTC 20962 trial, the median survival time was 46 months and 2 year survival rate was $69 \% .^{28} \mathrm{~A}$ report by Ishikawa et al. ${ }^{32}$ published in 
2003 also indicated much better survival rates for PCNSL patients treated by radiation alone, as compared to older published data.

Data for PCNSL patients seen between 2000 and 2004 have been published. ${ }^{22}$ Data for 131 patients were collected; $76 \%$ received chemotherapy and $72 \%$ of the chemotherapy regimens were high-dose MTX-based. As a result, 31 patients treated with radiation alone had a 5 -year survival rate of only $7.5 \%$. Furthermore, newer data for patients seen between 2005 and 2009 have recently been accrued and analyzed, and results will be published in due time. However, this review includes preliminary data on the survival of patients seen in 2005-2009 in comparison with data from the previous surveys. Patients' and tumor characteristics for the periods 1985-1994, 1995-1999, 2000-2004, and 20052009 are shown in Table 2. Treatment characteristics are shown in Table 3. In Figure 1, survival curves for all patients treated during the four periods are shown. The overall survival rates at 5 years were $15.3 \%, 29.5 \%, 30.4 \%$ and $43.6 \%$ for the periods of $1985-1994,1995-1999$, 2000-2004 and 2005-2009, respectively. The high survival rate in the most recent period may partly be due to a shorter follow-up period. Also, survival curves for patients treated by radiation alone are shown in Figure 2 . Only $23 \%$ of the patients received radiation therapy alone during 2005-2009 achieving a 3-year survival rate of 30.6\%. Thus, old data on radiation therapy alone do not represent the true outcome obtained by radiation monotherapy. The Japanese data for patients treated between 1990 and 1999 (i.e. 18\% 5-year survival) may be closer to the expected prognosis of PCNSL patients treated with radiation therapy alone.

\section{Optimal treatment volume}

Whole-brain irradiation has been commonly used to treat PCNSL patients. The rationale for using a whole-brain field is that PCNSL is often multiple and that the boundary of PCNSL lesions is obscure; tumor cells are believed to usually invade the normal brain over a few centimeters from a contrast-enhanced tumor mass. Therefore, wholebrain irradiation is safe in terms of not missing viable tumor cells. This was especially true in the era before MRI or CT. Following the traditional use of whole-brain fields, the majority of PCNSL patients are still being treated with whole-brain radiation. On the other hand, medical oncologists have stressed the toxicity of whole-brain radiation. The neurotoxicity includes dementia and a decline in PS. The neurotoxicity is more specific to the treatment of PCNSL patients, since we observed only a slight, if any, decline in the mini-mental state examination score after whole-brain irradiation in patients with brain metastases from other cancers. ${ }^{33}$ It could be speculated that PCNSL grows invasively against normal brain tissue so that such normal cells, even if they retain their function on diagnosis of PCNSL, are vulnerable to radiation. On the other hand, brain metastases and other tumors invade normal tissue less aggressively and normal cells are considered

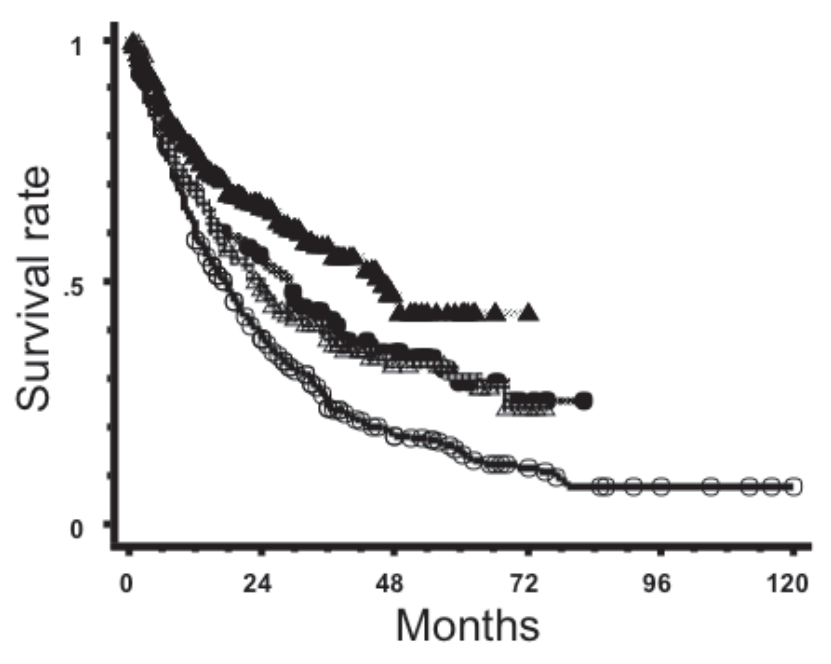

Figure 1. Survival curves for primary central nervous system lymphoma patients treated during 1985-1994 $(O, n=466)$, 1995$1999(\bullet, n=142), 2000-2004(\triangle, n=131)$ and 2005-2009 $(\Delta$, $\mathrm{n}=304)$.

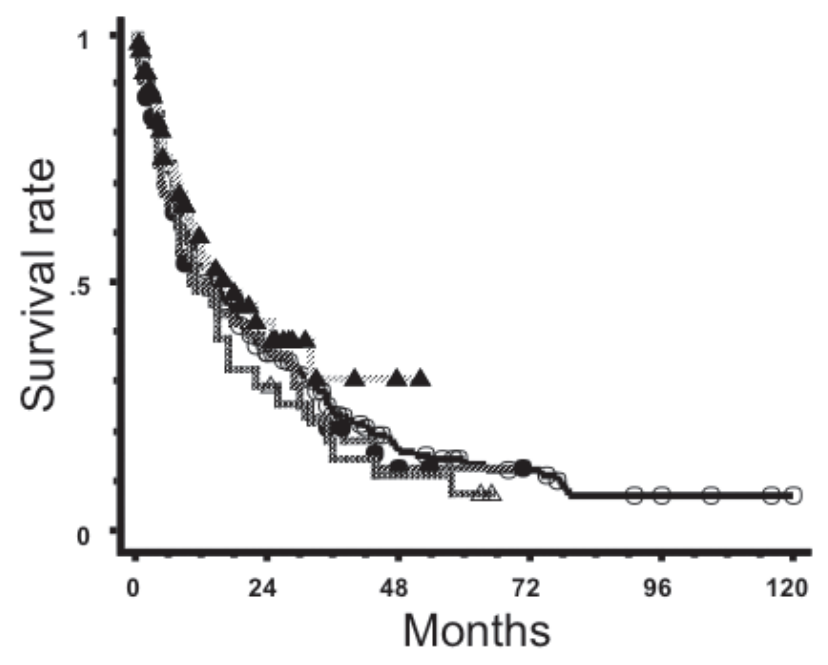

Figure 2. Survival curves for primary central nervous system lymphoma patients treated by radiotherapy alone during 1985-1994 $(\circ, \mathrm{n}=254), 1995-1999(\bullet, \mathrm{n}=55), 2000-2004(\triangle, \mathrm{n}=32)$ and 2005-2009 $(\Delta, \mathrm{n}=69)$.

Table 2. Changes in patient and tumor characteristics during 1985-2009.

\begin{tabular}{|c|c|c|c|c|c|}
\hline \multicolumn{2}{|l|}{ Characteristic } & \multicolumn{4}{|c|}{ Period (year) } \\
\hline & & 1985-1994 & 1995-1999 & $2000-2004$ & $2005-2009$ \\
\hline Gender & Male/total (\%) & $276 / 466(59)$ & $96 / 142(68)$ & $67 / 131(51)$ & 182/304 (60) \\
\hline Age (years) & Median (range) & $60(5-86)$ & $59(15-93)$ & $65(30-90)$ & $68(17-85)$ \\
\hline Performance status & $3,4(\%)$ & $209 / 438(48)$ & $55 / 138(40)$ & $37 / 128(29)$ & $75 / 301(25)$ \\
\hline Tumor number & Multiple (\%) & $175 / 460(38)$ & $56 / 140(40)$ & $72 / 131(55)$ & $157 / 304(52)$ \\
\hline Tumor size $(\mathrm{cm})$ at diagnosis & Mean \pm SD & $3.8 \pm 1.4$ & $3.8 \pm 1.6$ & $3.8 \pm 1.2$ & $3.6 \pm 1.4$ \\
\hline CSF dissemination & $+(\%)$ & $56 / 422(13)$ & 23/122 (19) & $20 / 126(16)$ & $28 / 300(9.3)$ \\
\hline
\end{tabular}

SD, standard deviation; CSF, cerebrospinal fluid. 
Table 3. Changes in treatment during 1985-2009.

\begin{tabular}{|c|c|c|c|c|c|}
\hline \multicolumn{2}{|c|}{ Characteristic } & \multicolumn{3}{|c|}{ Period (year) } & $2005-2009$ \\
\hline Surgery & Biopsy (\%) & - & $71 / 142(50)$ & 83/131 (63) & 234/303 (77) \\
\hline Brain radiation field & Partial brain (\%) & $37 / 466(7.9)$ & $12 / 142(8.5)$ & $5 / 131(11)$ & 21/304 (6.9) \\
\hline Spinal radiation & $+(\%)$ & $37 / 445(8.3)$ & 8/142 (5.6) & 4/131 (3.1) & $5 / 304(1.6)$ \\
\hline Total dose (Gy) & Mean \pm SD & $48.4 \pm 11.2$ & $48.7 \pm 10.8$ & $47.0 \pm 9.0$ & $46.9 \pm 8.8$ \\
\hline Iv chemotherapy & $+(\%)$ & $212 / 420(50)$ & $87 / 142(61)$ & $99 / 131(76)$ & 235/304 (77) \\
\hline MTX-containing regimen & $+(\%)$ & 47/212 (22) & 27/87 (31) & $71 / 99(72)$ & 196/235 (83) \\
\hline It chemotherapy & $+(\%)$ & $42 / 415(10)$ & 16/142 (11) & 8/131 (6.1) & 39/304 (13) \\
\hline
\end{tabular}

SD, standard deviation; Iv, intravenous; MTX, methotrexate; It, intrathecal.

to be more resistant to the adverse effects of radiation. However, our experience with Japanese patients shows that this neurotoxicity is not so frequently encountered in those under 60 years of age treated mainly with radiation therapy, and I think this adverse event is also closely related to the use of MTX. I suggest that neurotoxicity, when treated by radiation alone, might have been somewhat exaggerated by Western neuro-oncologists. However, this possible diversity in neurotoxicity may be due to racial differences and to our policy concerning radiation therapy, especially with respect to the treatment volume, as described below.

Therefore, the concern over neurotoxicity seems to be strengthened when chemotherapy, especially high-dose MTX, is used in combination. On the other hand, one may expect high-dose MTX to eradicate microscopic diseases, so I have postulated the use of extended focal radiation field with wide (approx. $4 \mathrm{~cm}$ ) margins from a tumor mass when there is a single PCNSL lesion. This is especially true when chemotherapy including high-dose MTX is used. After $40 \mathrm{~Gy}$, I reduce the treatment volume and use a localized radiation field with 1-1.5 cm margins. We retrospectively analyzed 41 patients treated by a partial-brain radiation field. ${ }^{26}$ When patients were treated with a localized field with less than 4 -cm margins, out-of-field recurrences were frequently seen $(83 \%$ at 3 years). With wider margins $(\geq 4 \mathrm{~cm})$, the rate for out-of-field recurrence was $22 \%$ at three years, and there did not seem to be any difference in overall survival to that of patients receiving whole-brain radiation therapy. Therefore, it was concluded that partial-brain irradiation with wide margins may well be worth considering in patients with a single lesion undergoing high-dose MTX-containing chemotherapy. We are still continuing to use extended focal radiation fields for patients with a single lesion. Figure 3 shows survival curves for PCNSL patients after whole-brain or partial-brain irradiation in the above-mentioned Japanese surveys. All patients treated between 1985 and 2009 were included. Patients treated with partial-brain fields had a 5 -year survival rate of $33.6 \%$, while those treated with whole-brain fields had a 5 -year survival of $24.4 \%(\mathrm{P}=0.47)$. A recent analysis of our own data also suggested results similar to those we published previously. ${ }^{26}$ Even when whole-brain radiation is employed, the whole-brain should not be irradiated with a full dose. After 40 or $30 \mathrm{~Gy}$ (depending upon the case), the radiation field should be reduced to only cover regions of macroscopic tumors confirmed at diagnosis with a margin. There is no rationale for using the same dose for macroscopic lesions and prophylaxis for possible microscopic lesions. Using whole-brain fields throughout the treatment should be avoided.

Craniospinal irradiation has not been frequently used in the treatment of PCNSL. Although the JASTRO surveys of PCNSL patients seen between 1985 and 2009 indicated that the incidence of meningeal dissemination at the diagnosis of PCNSL was 13-19\%, spinal irradiation was employed in only $3.1-8.3 \%$ of the patients. ${ }^{22,29-31}$ Figure 4 shows survival curves for PCNSL patients treated with or without spinal irradiation in the above-mentioned surveys. Patients who did not receive

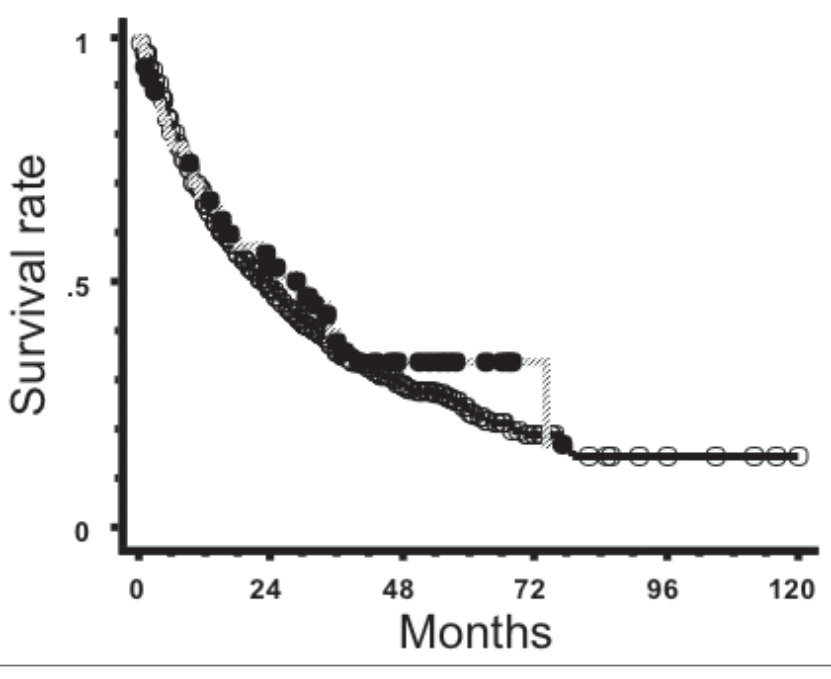

Figure 3. Survival curves for primary central nervous system lymphoma patients treated with whole-brain radiation $(\circ, \mathrm{n}=958)$ and those treated with partial-brain radiation $(\bullet, n=85)$.

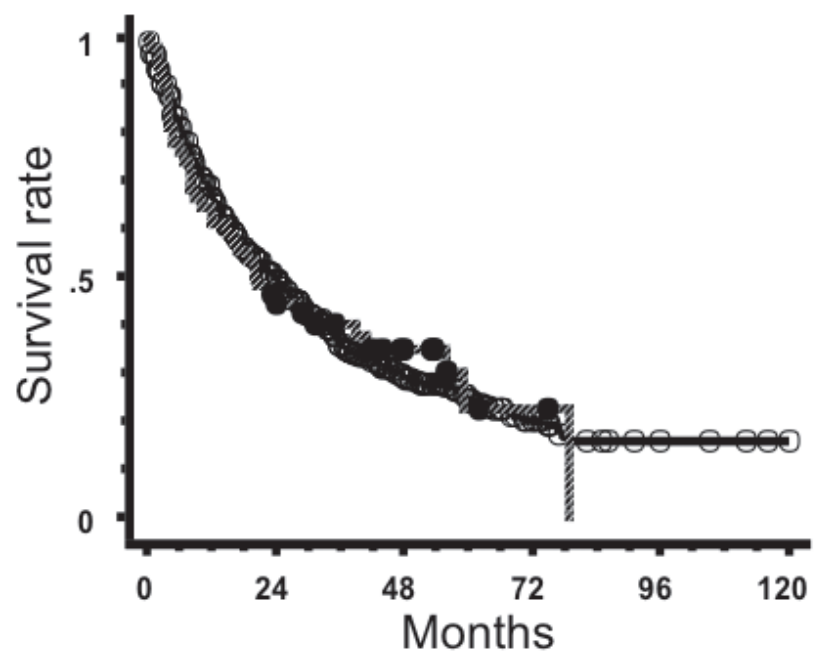

Figure 4. Survival curves for primary central nervous system lymphoma patients receiving no spinal irradiation $(\circ, n=964)$ and those receiving spinal irradiation $(\bullet, n=54)$ during $1985-2009$. 
whole spinal irradiation had a 5 -year survival rate of $25.5 \%$, while those who did had a rate of $22.6 \%(\mathrm{P}=0.96)$.

\section{Optimal radiation dose and fractionation}

Conventional fractionation has been used in the treatment of PCNSL. Since normal brain tissue is considered to have a low $\alpha / \beta$ ratio of approximately 2-3 Gy, it is generally not recommended to use a high dose per fraction. Except for palliative treatment, a 2-Gy daily fraction remains the standard. Accelerated fractionation or accelerated hyperfractionation may be another option for rapidly growing tumors. One small phase I study investigated accelerated hyperfractionation using 1.5 Gy twice daily to a total whole-brain dose of $45 \mathrm{~Gy}$ in combination with chemotherapy with high-dose MTX, idarubicin, dexamathazone, cytosine arabinoside, and cisplatin. ${ }^{34}$ Bromodeoxyuridine infusion was delivered 2-3 days prior to whole-brain radiotherapy and then weekly during radiotherapy. In the 12 patients studied, disease control was modest, but neurotoxicity was found to be unacceptable. It is not clear whether this neurotoxicity was related to the use of accelerated hyperfractionation or bromodeoxyuridine infusion. Another small pilot study of 10 patients investigated 2 Gy twice daily to a whole-brain dose of 50 Gy delivered over only 13 days without weekend interruptions. ${ }^{35}$ Nine of the 10 patients died and the median survival time was only 17 months. The authors concluded that the accelerated schedule of radiation therapy was ineffective and was associated with unacceptable toxicity. Hyperfractionation using 1.0-1.2 Gy twice daily has also been evaluated in a few studies. To reduce late CNS toxicity, the use of a lower dose per fraction is felt worthy of investigation. In the Radiation Therapy Oncology Group (RTOG) study 9310 investigating pre-radiation chemotherapy including MTX, vincristine and procarbazine, the radiation dose and fractionation were changed from 45 Gy in 25 fractions to 36 Gy with 1.2 Gy twice daily over three weeks. ${ }^{36}$ Although the hyperfractionation schedule represented a $25 \%$ reduction in biologically effective tumor dose as compared to the standard fractionation, progression-free survival and overall survival were not significantly affected. The hyperfractionation regimen delayed leukoencephalopathy but did not eliminate severe neurotoxicity from chemoradiation. Another study of 30 patients used hyperfractionated whole-brain radiation to 45 Gy with 1.0 Gy twice daily in combination with MTX $\left(8 \mathrm{~g} / \mathrm{m}^{2}\right)$, cytarabine and thiotepa followed by autologous stem cell transplantation. ${ }^{37}$ The 5-year overall survival probability was as high as $69 \%$ for all patients and $87 \%$ for 23 patients who received high-dose chemotherapy and autologous stem-cell transplantation. Five of the 30 patients (17\%) developed leukoencephalopathy, and this rate was evaluated as moderate compared with data in the literature. From these studies, hyperfractionated radiotherapy seems to deserve further investigation. The use of hyperfractionation was reported in another small study of 8 patients using a whole-brain dose of as high as 64.8 Gy in 54 fractions over 5.5 weeks. ${ }^{38}$ Six patients showed neurological deterioration without evidence of tumor recurrence, and the marked toxicity prompted study closure.

No randomized trial has been carried out regarding the optimal total radiation dose. With 41 patients, the RTOG 8315 study investigated an escalation of the total dose to $60 \mathrm{~Gy}$ ( $40 \mathrm{~Gy}$ to the whole brain followed by a 20-Gy focal boost), but the results were not considered superior to those obtained by lower doses of radiation (e.g. 50 Gy). 5,36 Thus, the standard radiation dose in the primary treatment of PCNSL, when used alone, seems to remain at 50 Gy. With the development of MTX-containing chemotherapy, however, attempts are being made to reduce radiation doses (or even defer radiotherapy). In a phase II study with 57 patients, however, the reduction of the whole-brain dose from 45 to 30.6
Gy appeared to be associated with an increased recurrence rate. ${ }^{39} 0$ n the other hand, in a phase II study enrolling 30 patients, only 23.4 Gy were given to patients who achieved a complete response after chemotherapy, and the disease control was reported to be satisfactory. ${ }^{40}$ Figure 5 shows survival data from the above-mentioned Japanese surveys according to the total radiation dose. Patients who did not complete the planned radiotherapy were excluded. The 5 -year survival rate was $21.9 \%$ for patients receiving $30-39.9 \mathrm{~Gy}, 28.8 \%$ for those receiving 40-49.9 Gy, $27.0 \%$ for those treated with 50-53.9 Gy, and $21.0 \%$ for those receiving 54 Gy or more. Significant differences were observed between the lowest dose group and the other three groups. Patients receiving 40-49.9 Gy had higher survival rates than those receiving 54 Gy or more $(\mathrm{P}=0.039)$. Although these results were obtained retrospectively, they did not suggest a better outcome when treated with relatively low radiation doses, even in combination with chemotherapy.

As stated above, some groups are investigating the omission of radiation therapy in elderly patients. Even in younger patients, an attempt is being made to defer radiation and reserve it until recurrence. To examine this, a randomized trial was carried out by the German Primary CNS Lymphoma Study Group involving 551 patients. ${ }^{25}$ The trial compared high-dose MTX alone with high-dose MTX plus whole-brain radiotherapy to $45 \mathrm{~Gy}$ given as 1.5 Gy once daily. In the group treated without whole-brain radiation, high-dose cytarabine was administered when a complete response was not obtained by high-dose MTX. Also, this group of patients could receive radiation therapy at recurrence. The results clearly showed higher progression-free survival rates in the group treated with chemotherapy plus whole-brain radiation, but there was no significant difference in overall survival between the two groups; it seems that radiation given as second-line treatment was partially responsible for this result. Several weak points about this trial have been pointed out, including a long patient accrual period (9 years), a low rate of patients treated per protocol, and low overall survival rates in both arms. Therefore, future studies should examine this issue more closely, regardless of whether or not the omission or deferral of radiation therapy is really justified.

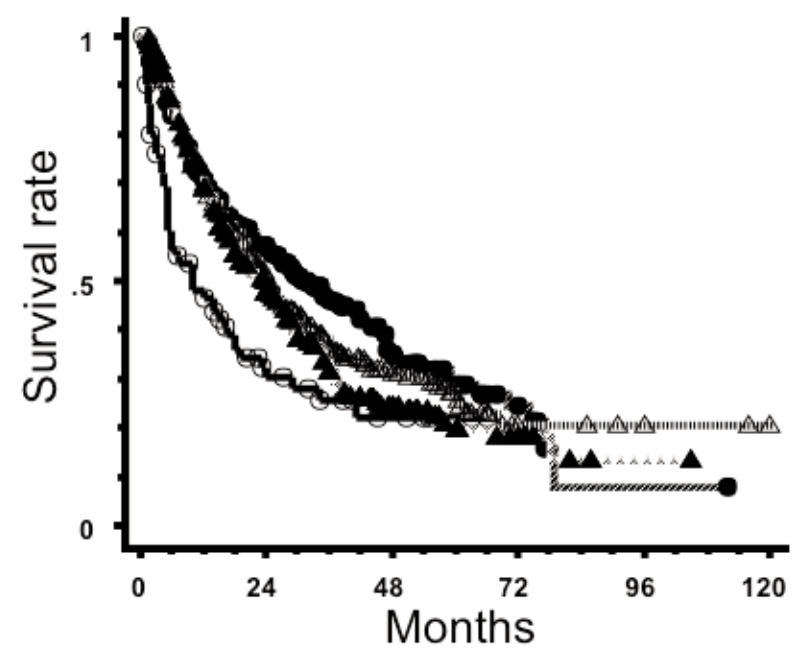

Figure 5. Survival curves for primary central nervous system lymphoma patients treated with a total dose of 30-39.9 Gy $(\circ, n=81)$, those treated with 40-49.9 $\mathrm{Gy}(\bullet, \mathrm{n}=272)$, those treated with 50 53.9 Gy $(\triangle, \mathrm{n}=356)$ and those treated with 54 Gy or more $(\Delta$, $\mathbf{n}=291)$. 


\section{Newer radiation technique and modalities}

Some groups have reported the use of stereotactic radiosurgery in the treatment of PCNSL. ${ }^{41,42}$ Its use in recurrent cases that have already undergone conventional full-dose radiation may be partly justified, but considering the spread of tumor cells around enhancing tumor masses, it is not sensible to use radiosurgery in the first-line treatment of PCNSL. Recurrent cases already receiving full-dose radiation and primary cases that are unable to undergo standard radiotherapy may be palliatively treated with a gammaknife, cyberknife, tomotherapy, or a linac-based radiosurgery system. Radiation doses may be determined on a case-by-case basis. The results of radiosurgical treatment for recurrent PCNSL indicate acceptable toxicity and a modest survival time. ${ }^{41,42}$ As expected, local control was relatively favorable, but high recurrence rates outside the treatment volume have been reported.

As a first-line treatment, intensity-modulated radiation therapy (IMRT) deserves consideration. In the treatment of glioblastoma, the simultaneous integrated boost technique is being increasingly used. ${ }^{43,44}$ If this technique is used, whole-brain or margin areas for focal radiation with generous margins can be uniformly treated with a standard dose, while tumor mass areas can be simultaneously boosted. For example, the whole brain or wide-margin regions around a tumor mass can be treated with 40 Gy in 20 fractions, while tumor masses can be treated with 2.4 or $2.5 \mathrm{~Gy}$ per fraction to the same 20 fractions. In my opinion, this type of IMRT should in the near future be employed in as many patients with primary malignant brain tumors as possible.

The use of particle beams has not yet been reported. The advantage of proton beams that stop at the target can be utilized for focal radiotherapy or as a boost. So, with the increase in the number of devices available, the use of proton therapy may be investigated in the future. On the contrary, carbon-ion therapy may not be indicated for PCNSL at present in view of the relatively high CNS toxicity reported in patients treated with carbon-ion beams. ${ }^{45}$

\section{Conclusions}

As time passes, the role of radiation therapy in the treatment of PCNSL may be diminishing and medical neuro-oncologists have led this trend. Radiation has a greater effect on local tumor control than chemotherapy. Therefore, the utilization of radiation therapy, even at lower doses than previously used, should be investigated in future studies, especially in younger patients, in order to cure this disease. Randomized trials should be encouraged to investigate this.

\section{References}

1. Littman P, Wang CC. Reticulum sarcoma of the brain. A review of the literature and a study of 19 cases. Cancer 1975;35:1412-20.

2. Leibel SA, Sheline GE. Radiation therapy for neoplasms of the brain. J Neurosurg 1987;66:1-22.

3. Shibamoto Y, Tsutsui K, Dodo Y, et al. Improved survival rate in primary intracranial lymphoma treated by high-dose radiation and systemic vincristine-doxorubicin-cyclophosphamide-prednisolone chemotherapy. Cancer 1990;65:1907-12.

4. Watne K, Scott H, Hager B, et al. Primary malignant lymphoma of the brain. A report of 24 cases from the Norwegian Radium Hospital. Acta Oncol 1992;31:545-50.

5. Nelson DF, Martz KL, Bonner H, et al. Non-Hodgkin's lymphoma of the brain: can high dose, large volume radiation therapy improve survival? Report on a prospective trial by the Radiation Therapy Oncology Group (RTOG): RTOG 8315. Int J Radiat Oncol Biol Phys 1992;23:9-17.

6. Hayabuchi N, Shibamoto Y, Nakamura K, et al. Stage I and II aggressive B-cell lymphomas of the head and neck: radiotherapy alone as a treatment option and the usefulness of the new prognostic index B-ALPS. Int J Radiat Oncol Biol Phys 2003;55:44-50.

7. Martinet S, Ozsahin M, Belkacemi Y, et al. Outcome and prognostic factors in orbital lymphoma: a Rare Cancer Network study on 90 consecutive patients treated with radiotherapy. Int J Radiat Oncol Biol Phys 2003;55:892-8.

8. Schultz C, Scott C, Sherman W, et al. Preirradiation chemotherapy with cyclophosphamide doxorubicin, vincristine, and dexamethazone for primary CNS lymphomas: initial report of Radiation Therapy Oncology Group protocol 88-06. J Clin Oncol 1996;14:55664.

9. 0'Neill BP, O'Fallon JR, Earle JD, et al. Primary central nervous system non-Hodgkin's lymphoma (PCNSL): survival advantages with combined initial therapy? A final report of the North Central Cancer Treatment Group (NCCTG) study 86-72-52. Int J Radiat Oncol Biol Phys 1999;43:559-63.

10. Shibamoto Y, Sasai K, Oya N, et al. Systemic chemotherapy with vincristine, cyclophosphamide, doxorubicin and prednisolone following radiotherapy for primary central nervous system lymphoma: a phase II study. J Neurooncol 1999;42:161-7.

11. Mead GM, Bleehen NM, Gregor A, et al. A Medical Research Council randomized trial in patients with primary cerebral non-Hodgkin lymphoma. Cerebral radiotherapy with and without cyclophosphamide, doxorubicin, vincristine, and prednisone chemotherapy. Cancer 2000;89:1359-70.

12. Glass J, Gruber ML, Chef L, et al. Preirradiation MTX chemotherapy of primary central nervous system lymphoma: long-term outcome. J Neurosurg 1994;81:188-95.

13. Blay JY, Conroy T, Chevreau C, et al. High-dose MTX for the treatment of primary cerebral lymphomas: analysis of survival and late neurologic toxicity in a retrospective series. J Clin Oncol 1998;16:864-71.

14. Brada M, Hjiyiannakis D, Hines F, et al. Short intensive primary chemotherapy and radiotherapy in sporadic primary CNS lymphoma. Int J Radiat Oncol Biol Phys 1998;40:1157-62.

15. Ferreri AJM, Reni M, Villa E. Therapeutic management of primary central nervous system lymphoma: lessons from prospective trials. Ann Oncol 2000;11:927-37.

16. Abrey LE, Yahalom J, DeAngelis LM. Treatment for primary CNS lymphoma: the next step. J Clin Oncol 2000;18:3144-50.

17. Reni M, Ferreri AJ, Guha-Thakurta N, et al. Clinical relevance of consolidation radiotherapy and other main therapeutic issues in primary central nervous system lymphomas treated with upfront high-dose MTX. Int J Radiat Oncol Biol Phys 2001;51:419-25.

18. Calderoni A, Aebi S. Combination chemotherapy with high-dose MTX and cytarabine with or without brain irradiation for primary central nervous system lymphomas. J Neurooncol 2002;59:227-30.

19. DeAngelis LM, Seiferheld W, Schold SC, et al. Combination chemotherapy and radiotherapy for primary central nervous system lymphoma: Radiation Therapy Oncology Group study 93-10. J Clin Oncol 2002;20:4643-8.

20. Poortmans PMP, Kluin-Nelemans HC, Haaxma-Reiche H, et al. High-dose methotrexate-based chemotherapy followed by consolidating radiotherapy in non-AIDS-related primary central nervous system lymphoma: European Organization for Research and Treatment of Cancer Lymphoma Group Phase II trial 20962. J Clin Oncol 2003;21:4483-8.

21. Bessel EM, Graus F, Lopez-Guillermo A, et al. Primary non- 
Hodgkin's lymphoma of the CNS treated with CHOD/BVAM or BVAM chemotherapy before radiotherapy: long-term survival and prognostic factors. Int J Radiat Oncol Biol Phys 2004;59:501-8.

22. Shibamoto Y, Ogino H, Suzuki G, et al. Primary central nervous system lymphoma in Japan: changes in clinical features, treatment and prognosis during 1985-2004. Neuro Oncol 2008;10:560-8.

23. Rubin P, Gashu DM, Hansen JT, et al. Disruption of the blood-brain barrier as the primary effect of CNS irradiation. Radiother Oncol 1994;31:51-60.

24. DeAngelis LM. Primary central nervous system lymphoma: a curable brain tumor. J Clin Oncol 2004;24:4471-3.

25. Thiel E, Korfel A, Martus P, et al. High-dose methotrexate with or without whole brain radiotherapy for primary CNS lymphoma (GPCNSL-SG-1): a phase 3, randomized, non-inferiority trial. Lancet Oncol 2010;11:1036-47.

26. Shibamoto Y, Hayabuchi N, Hiratsuka J, et al. Is whole-brain irradiation necessary for primary central nervous system lymphoma? Patterns of recurrence following partial-brain irradiation. Cancer 2003;97:128-33.

27. Shibamoto Y, Sasai K, Oya N, et al. Intracranial germinoma: radiation therapy with tumor volume-based dose selection. Radiology 2001;218:452-6.

28. Shibamoto $\mathrm{Y}$, Ogino $\mathrm{H}$, Hasegawa $\mathrm{M}$, et al. Results of radiation monotherapy for primary central nervous system lymphoma in the 1990's. Int J Radiat Oncol Biol Phys 2005;62:809-13.

29. Hayabuchi N, Shibamoto Y, Onizuka Y, et al. Primary central nervous system lymphoma in Japan: a nationwide survey. Int J Radiat Oncol Biol Phys 1999;44:265-72.

30. Shibamoto Y, Tsuchida E, Seki K, et al. Primary central nervous system lymphoma in Japan 1995-1999: changes from the preceding 10 years. J Cancer Res Clin Oncol 2004;130:351-6.

31. Kawamura T, Ishiguchi T, Shibamoto Y, et al. Results of primary central nervous system lymphoma treated by radiation and chemotherapy: retrospective analysis of twelve institutions in the Tokai district in Japan, 1995-1999. Radiat Med 2006;24:9-16.

32. Ishikawa H, Hasegawa M, Tamaki Y, et al. Comparable outcomes of radiation therapy without high-dose methotrexate for patients with primary central nervous system lymphoma. Jpn J Clin Oncol 2003;33:443-9.

33. Shibamoto Y, Baba F, Oda K, et al. Incidence of brain atrophy and decline in mini-mental state examination score after whole-brain radiotherapy in patients with brain metastases: a prospective study. Int J Radiat Oncol Biol Phys 2008;72:1168-73.
34. Dabaja BS, McLaughlin P, Ha CS, et al. Primary central nervous system lymphoma: Phase I evaluation of infusional bromodeoxyuridine with whole brain accelerated fractionation radiation therapy after chemotherapy. Cancer 2003;98:1021-8.

35. Laperriere NJ, Wong CS, Milosevic MF, et al. Accelerated radiation therapy for primary lymphoma of the brain. Radiother Onco 1998;47:191-5.

36. Fisher B, Seiferheld W, Schultz C, et al. Secondary analysis of Radiation Therapy Oncology Group study (RTOG) 9310: an intergroup phase II combined modality treatment of primary central nervous system lymphoma. J Neurooncol 2005;74:201-5.

37. Illerhaus G, Marks R, Ihorst G, et al. High-dose chemotherapy with autologous stem-cell transplantation and hyperfractionated radiotherapy as first-line treatment of primary CNS lymphoma. J Clin Oncol 2006;24:3865-70.

38. Davey P, Catton C, Ngan B, et al. Phase I study of hyperfractionated whole brain irradiation (HWBI) in the treatment of primary cerebral lymphoma. Clin Oncol 1993;5:159-64.

39. Bessell EM, Lopez-Guillermo A, Villa S, et al. Importance of radiotherapy in the outcome of patients with primary CNS lymphoma: an analysis of the CHOD/BVAM regimen followed by two different radiotherapy treatments. J Clin Oncol 2002;20:231-6.

40. Shah GD, Yahalom J, Correa DD, et al. Combined immunochemotherapy with reduced whole-brain radiotherapy for newly diagnosed primary CNS lymphoma. J Clin Oncol 2007;25:4730-5.

41. Kenai H, Yamashita M, Nakamura T, et al. Gamma Knife surgery for primary central nervous system lymphoma: usefulness as palliative local tumor control. J Neurosurg 2006;105:133-8.

42. Sakamoto M, Oya N, Mizowaki T, et al. Initial experiences of palliative stereotactic radiosurgery for recurrent brain lymphomas. J Neurooncol 2006;77:53-8.

43. Suzuki M, Nakamatsu K, Kanamori S, et al. Feasibility study of the simultaneous integrated boost (SIB) method for malignant gliomas using intensity-modulated radiotherapy (IMRT). Jpn J Clin Oncol 2003;33:271-7.

44. Iuchi T, Hatano K, Narita Y, et al. Hypofractionated high-dose irradiation for the treatment of malignant astrocytomas using simultaneous integrated boost technique by IMRT. Int J Radiat Oncol Biol Phys 2006;64:1317-24.

45. Miyawaki D, Murakami M, Demizu Y, et al. Brain injury after proton therapy or carbon ion therapy for head-and-neck cancer and skull base tumors. Int J Radiat Oncol Biol Phys 2009;75:378-84. 